\title{
General Framework of Hearing Aid Fitting Management
}

\author{
Soo Hee Oh ${ }^{1}$ and Junghak Lee ${ }^{2}$ \\ ${ }^{1}$ Audiology Institute, ${ }^{2}$ Department of Audiology, Hallym University of Graduate Studies, Seoul, Korea
}

Received October 21, 2015

Revised November 26, 2015

Accepted November 30, 2015

\author{
Address for correspondence \\ Junghak Lee, FAAA, BCA, PhD \\ Department of Audiology, \\ Hallym University of Graduate Studies, \\ 427 Yeoksam-ro, Gangnam-gu, \\ Seoul 06197, Korea \\ Tel $+82-2-2051-4950$ \\ Fax +82-2-3453-6618 \\ E-mail leejh@hallym.ac.kr
}

\begin{abstract}
Hearing aids are one of the most widely used treatment options for the hearing impaired and optimal outcomes of hearing aids are supported by comprehensive hearing aid fitting protocols. Currently, the term 'hearing aid fitting' is prevalently used among service and industry sectors with its comprehensive procedures not systematically explicated. In addition, a variety of non-normalized guidelines for hearing aid fitting has led to non-uniform care, outcome variability, and dissatisfaction of the use of hearing aids. The main purpose of the present study is to suggest a general framework of standardized practice for hearing aid fitting management including its pre- and post-fitting stages. The management framework centers on its fitting process with its prior steps of assessment as well as its posterior steps of followup, thereby eliminating diverging interpretations and non-uniform practices. Outcomes of this study are also expected to improve potential benefits such as quality of hearing aid fitting, user satisfaction, and cost effectiveness across relevant stakeholders.
\end{abstract}

J Audiol Otol 2016;20(1):1-7

KEY WORDS: Hearing aids · Hearing aid fitting management · Standardization.

\section{Introduction}

The world report on disability in 2012 estimated there are 360 million people with hearing loss representing, approximately $5.3 \%$ of the world population [1]. In recent years, aging has become one of the most common causes of hearing loss and the prevalence of hearing loss among adults over 65 years of age is five times higher than that among adults under 65 years of age [2]. According to the World Health Organization, an $18-50$ percentage increase in 65 years of age and over is expected from 2010-2020, which will lead to an increase in the hearing impaired [2].

As the number of people with hearing impairments has increased, treatments for hearing loss have become an important issue [2]. Hearing aids are one of the most widely-used treatment options for the hearing impaired [3-5]. For the proper use of hearing aids, hearing aid fitting management (HAFM) is a crucial issue for manufacturers, dispensers, and service

This is an Open Access article distributed under the terms of the Creative Commons Attribution Non-Commercial License (http://creativecommons. org/licenses/by-nc/3.0/) which permits unrestricted non-commercial use, distribution, and reproduction in any medium, provided the original work is properly cited. providers, and especially for hearing aid users [3,6,7]. Optimal outcomes of hearing aids are supported by comprehensive hearing aid fitting protocols [6] and the impact of "poor fit and comfort" is particularly notable for hearing aid return rates [7]. Accordingly, the whole process of hearing aid fitting needs to be normalized for its efficiency and transparency, user satisfaction, and cost effectiveness.

Two issues are expected to influence hearing aid markets and stakeholders, and lead to the exploration of the standardization of HAFM in this study. First, the term 'hearing aid fitting' is prevalently used among service and industry sectors with its comprehensive procedures not systematically explicated [8-10]. It has potentially conflicting interpretations and misunderstandings. Second, a variety of non-normalized guidelines for hearing aid fitting has led to non-uniform care, outcome variability, and dissatisfaction of the use of hearing aids [11-26].

The main purpose of the present study is to suggest a general framework of standardized practice for HAFM including the pre- and post-fitting stages. To establish the framework, this study investigated various guidelines and essential components for hearing aid fitting around the world. The management framework centers on its fitting process with its prior 
steps of assessment as well as its posterior steps of followup, thereby eliminating diverging interpretations and nonuniform practices. The outcomes of this study are expected to improve potential benefits such as quality of hearing aid fitting, user satisfaction, and cost effectiveness, for all relevant stakeholders.

\section{Essential Components of HAFM}

The establishment of HAFM relies on the extraction and unification of fundamental components from pre-existing hearing aid fitting guidelines for both adults and children [11-26]. This general framework includes all the necessary components for a best practice of HAFM. Table 1 summarizes the essential components of hearing aid fitting guidelines globally (one standard and 15 guidelines) consisting of 11 components: counseling, audiometry, hearing aid evaluation, hearing aid selection, ear impression, adjustment, verification, orientation, auditory training, outcome measures, and comprehensive report. It is evident that most of these components are common across the guidelines although each guideline includes slightly different components.

\section{General Framework of HAFM}

The HAFM in this study is defined as a systematic process targeting hearing aid fitting with its prior and posterior stages to help the hearing impaired recognize and interpret sounds better with hearing aids by providing auditory training as well as optimizing audibility and comfort. Fig. 1 shows the general framework of HAFM based on the documentary data of Table 1 with three inclusive aspects: functional, relational, and procedural. The functional aspect includes the use of hearing aids for helping people with hearing impairments by improving their hearing ability and overall satisfaction. The relational aspect specifies various relations involving hearing aids, as depicted in Fig. 1, with three modules: 1) assessment, 2) fitting, and 3) follow-up. The procedural aspect is modeled by a potential flow occurring in the process of HAFM and this model is simply depicted below: 1) Start at assessment, 2) Go to fitting, 3) Go to follow-up, 4) No further action required if satisfied, 5) Go to fitting if unsatisfied.

At the pre-fitting stage, the assessment module focuses on identifying hearing loss, functional problems, and hearing aid candidacy and selecting appropriate hearing aids. This module encompasses several components including counseling, audiometry testing, hearing aid evaluation, hearing aid selection, and ear impression.

The next stage of HAFM involves the fitting module which establishes optimal audibility and physical comfort with hearing aids while ensuring good sound quality. Ideally, the fitting module efficiently restores hearing loss based on types, degrees, and configurations of individual hearing by adjusting hearing aids. Then, optimal sound quality and comfort are verified with subjective and objective tests. Use and care instructions for hearing aids are also provided at this module.

At the post-fitting stage, the follow-up module focuses on maximizing and validating hearing aid benefits through auditory training and outcome measures, respectively. Auditory training attempts to maximize various effects associated with hearing ability, speech perception, and hearing functions of users. Outcome measures validate acoustic and psycho-social performances of users after a specified period of time of hearing aid adoption with or without auditory training. Finally, a comprehensive report for the whole HAFM is necessary for current and future references. As depicted in Fig. 1, the fitting module may be repeated if the follow-up module indicates unsatisfactory outcomes.

Taken together, the general framework of HAFM emphasizes three factors: 1) importance of three main modules, 2) interactive relations between the modules, and 3) systematic approaches to improve outcomes with hearing aids.

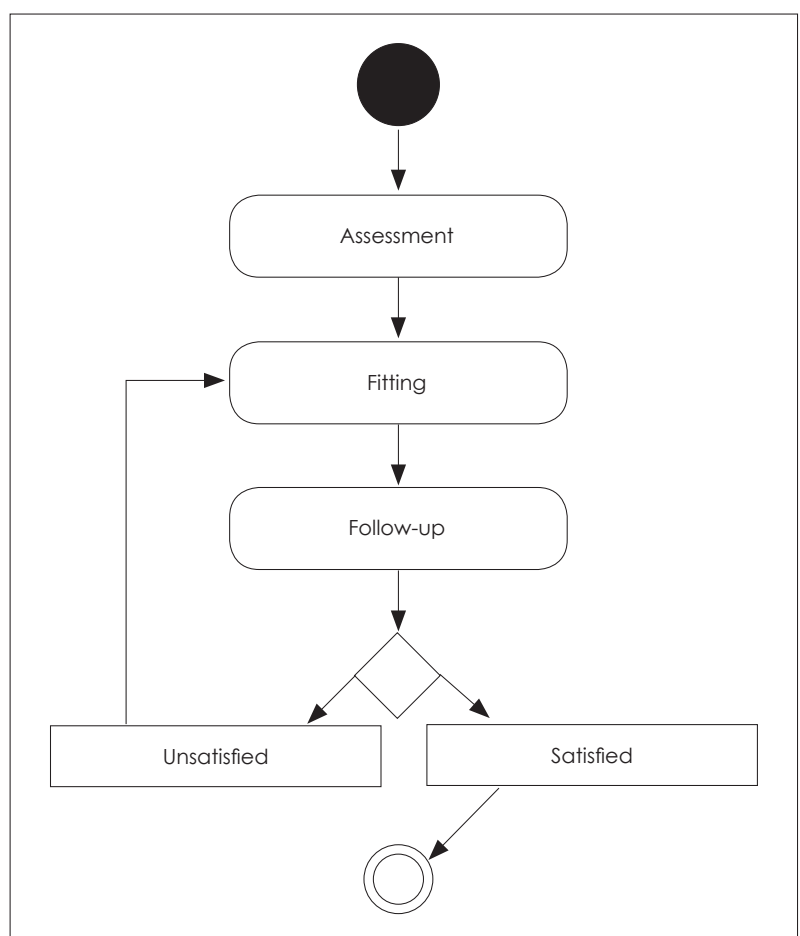

Fig. 1. General framework of hearing aid fitting management. The symbols in each figure (Fig.1-4) follow the unified modeling language [27]. 
Oh SH, et al.

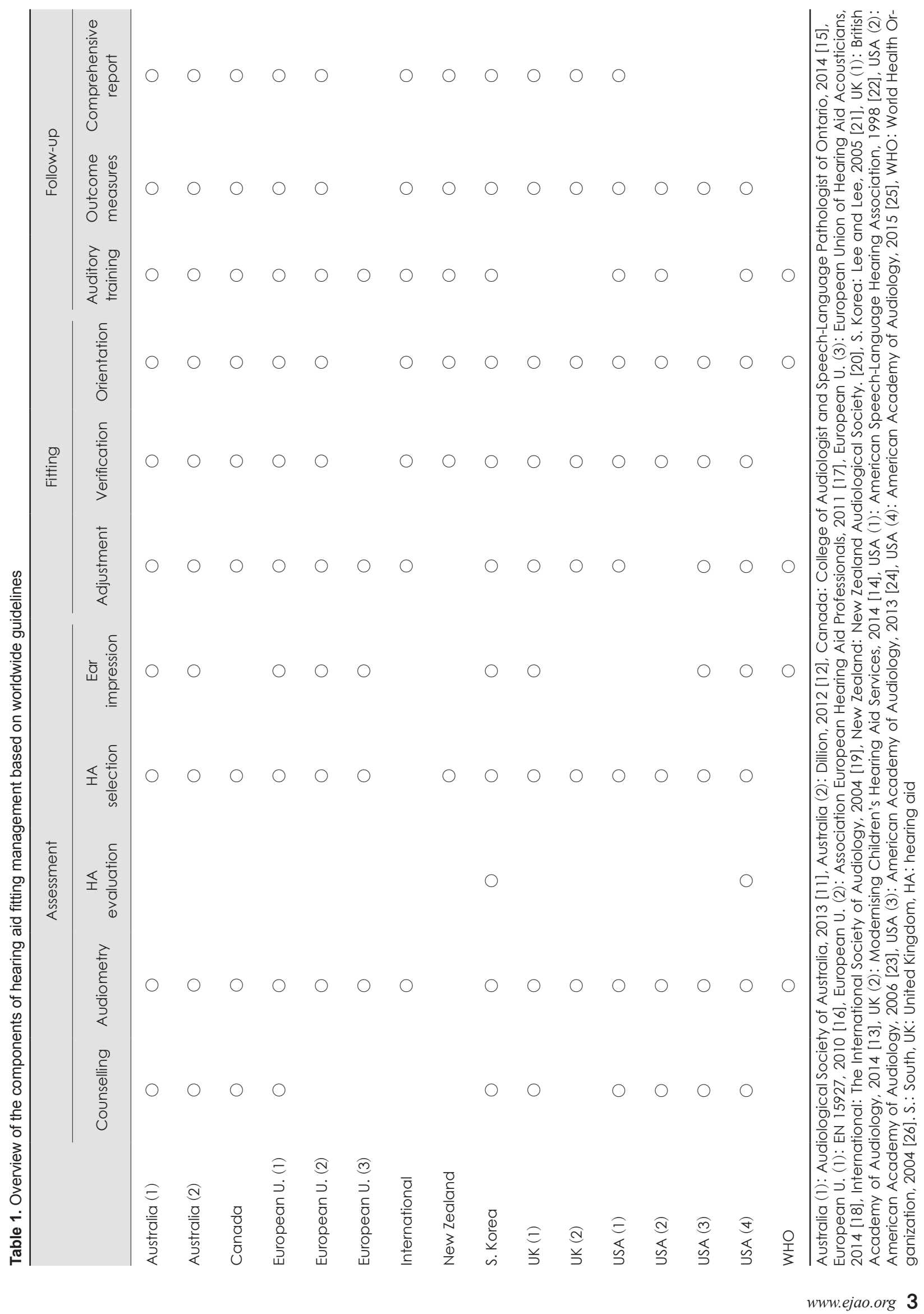




\section{Three Modules of HAFM}

\section{Assessment module}

Fig. 2 illustrates the assessment module consisting of five components: 1) counseling [11-13,15,16,19,21-25], 2) audiometry [11-19,21-26], 3) hearing aid evaluation [21,25], 4) hearing aid selection [11-18,20-25], and 5) ear impression [11-13,16-18,21,24-26]. The whole process of this module is simply depicted below: 1) Start at counseling, 2) Go to audiometry, 3) Go to hearing aid evaluation, 4) Go to hearing aid selection, 5) Go to ear impression if a customized type of hearing aid is selected, 6) Do not take ear impression if noncustomized type of hearing aid is selected.

The assessment module serves as a framework for investigating the pre-fitting stage that consists of preparatory components described below. It is generally accepted that counseling identifies individual needs, concerns, and abilities and establishes realistic expectations [11-13,15,16,19,21-25]. Emo-

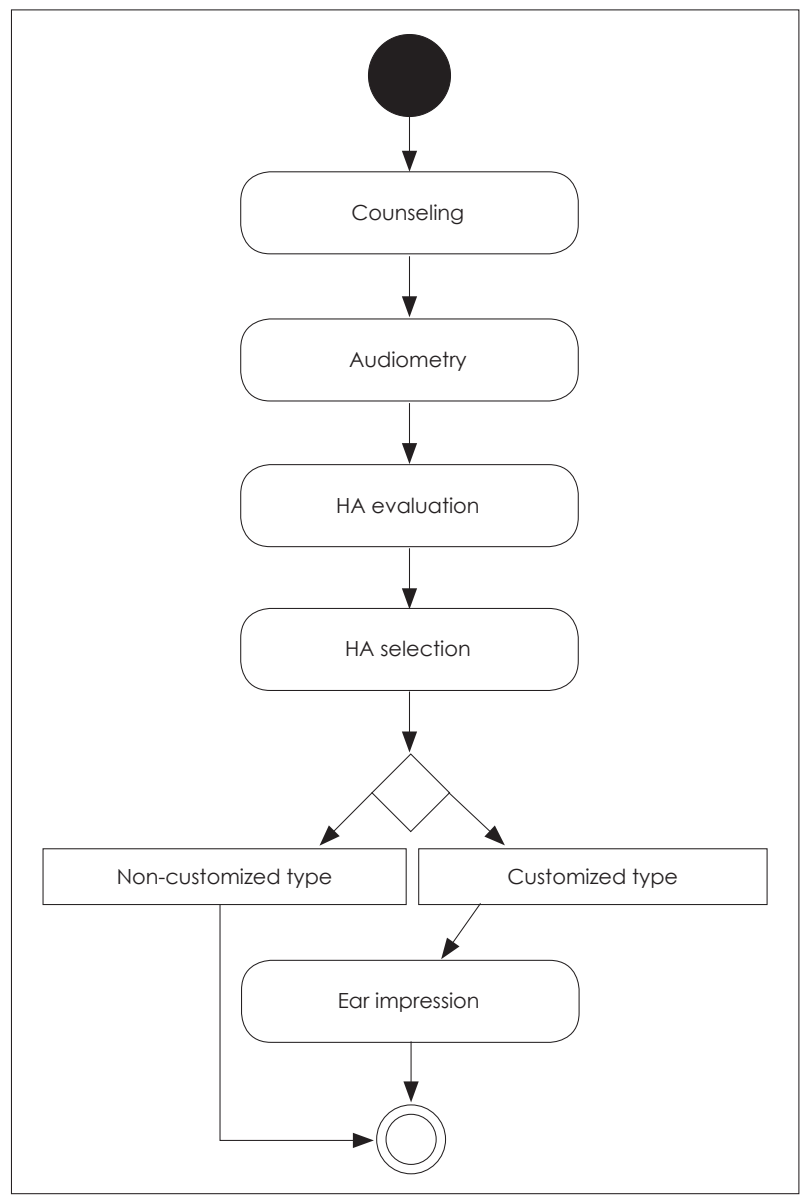

Fig. 2. Assessment module. Placing counseling on the first stage of this module does not indicate that counseling will be exclusively performed at the first stage of HAFM. Counseling component is inclusive across three modules although it was not shown in other figures. HAFM: hearing aid fitting management, HA: hearing aid. tional factors including anxiety control associated with hearing loss are also identified. Audiometry primarily evaluates hearing loss of potential hearing aid users by measuring hearing thresholds and speech recognition scores with various tests such as pure tone audiometry [11-19,21-26]. In most cases, hearing aid evaluation, pre-treatment trial, is performed with a loaner hearing aids and it attempts to establish realistic expectations by providing typical experiences of wearing hearing aids before making a decision of users' own hearing aids $[21,25]$. Hearing aid selection includes decision making processes associated with multiple features of hearing aids by considering device features, and anatomic characteristics and cosmetic factors of potential hearing aid users [11-18,20-25]. Specifically, it determines earmold, style, vent, and other physical and acoustical properties of hearing aids. Then, ear impression is taken if a customized type of hearing aid is selected, such as shell or behind-the-ear type [1113,16-18,21,24-26]. However, taking an ear impression is not necessary for a non-customized type of hearing aid.

\section{Fitting module}

Fig. 3 illustrates the fitting module consisting of three components: 1) adjustment [11-19,21,22,24-26], 2) verification [11-17,19-25], and 3) orientation [11-17,19-26]. The whole process of this module is simply depicted below: 1) Start at adjustment, 2) Go to verification, 3) Go to orientation if verified, 4) Go to adjustment if failed verification.

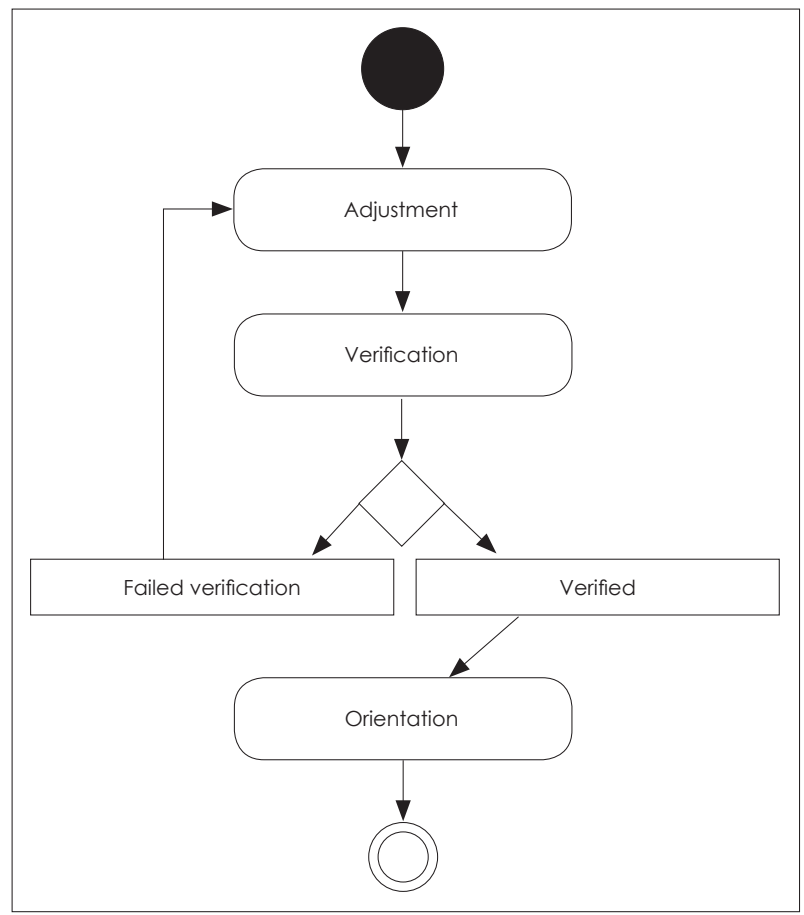

Fig. 3. Fitting module. 
Adjustment involves physical and electro-acoustic aspects [11-19,21,22,24-26]. Physical adjustment aims for maintaining the physical integrity and comfort in wear by modifying and checking physical features of hearing aids such as modification of earmolds or tubes and evaluation of physical adequacy. Electro-acoustic adjustment targets optimal establishments of electro-acoustic characteristics of hearing aids based on types, degrees, and configurations of individual hearing loss. For example, it involves applications of appropriate fitting formula and compression ratio.

Main contents of adjustment are: 1) Quality control: analysis of electro-acoustic specification [28], 2) Selecting a fitting formula based on individual audiogram, 3) Checking physical and electro-acoustic comfort (e.g., acoustic feedback, occlusion), 4) Modifying physical and electro-acoustic characteristics.

Verification, is a process evaluating physical, electro-acoustic, and psycho-acoustic aspects with hearing aids by presenting signals to hearing aids in the test box or a real-ear and evaluating subjective responses using questionnaires [1117,19-25]. Adjustment may be repeated in case of failed verification.

Main contents of verification are: 1) Coupler measurements [28], 2) Real ear measurements [29], 3) Sound field tests [30], 4) Psycho-acoustic checking associated with loudness comfort/discomfort, 5) Questionnaires focusing on acoustic aspects.

Orientation, the final component of the fitting module, mainly provides the information relevant to operational techniques, maintenance strategies including trouble shootings, realistic performance expectations, and other resources needed for hearing aid users [11-17,19-26].

\section{Follow-up module}

Fig. 4 presents the follow-up module consisting of three components: 1) auditory training [11,12,15-23,25,26], 2) outcome measures [11-17,19-25], and 3) comprehensive report [11-17,19-22]. The whole process of this module is simply depicted below: 1) Start at auditory training, 2) Go to outcome measures, 3) Go to comprehensive report if satisfied, and 4) Go to auditory training if unsatisfied.

The follow-up module serves as a framework for investigating the components of a post-fitting stage described below. Auditory training is an action to aid the capability of hearing perception through hearing aids and to maximize performance with hearing aids by focusing on various rehabilitative aspects including auditory perception, communication strategies, and individual needs [11,12,15-23,25,26]. Although entire training sessions depend on the capability and

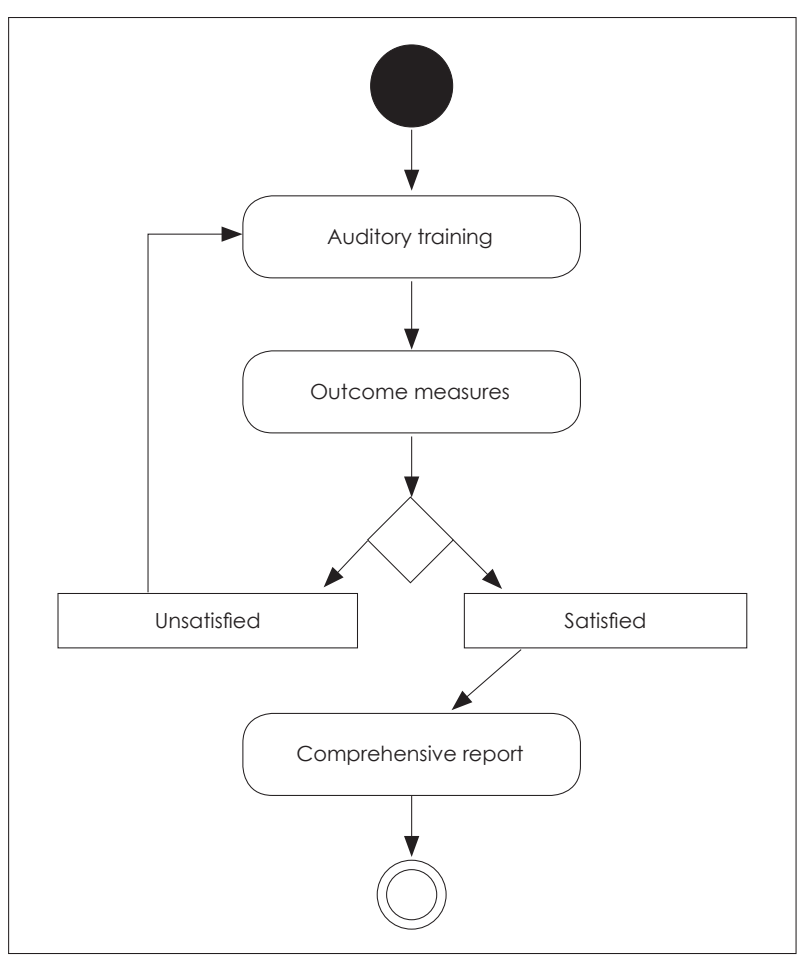

Fig. 4. Follow-up module.

need of individual hearing aid users, it is generally accepted that auditory training consists of consecutive training sessions for a certain amount of time.

Outcome measures validate multidimensional aspects of hearing aid usage by measuring the outcomes associated with acoustic and psycho-social aspects of hearing aid users [1117,19-25,31]. Some common outcome domains include measures of speech recognition performance and objective benefits with hearing aids and subjective measures of sound quality, listening efforts, hearing aid usage, and satisfaction [31]. As previously mentioned in Fig. 1, if the results from outcome measures with hearing aids are unsatisfactory, the fitting module may be repeated.

Comprehensive report reflecting substantial information occurred during the whole HAFM process is filed for informative reports and future references at the final stage of the module [11-17,19-22]. Additionally, it is important to note that documentations for each module and component are also needed although this framework does not specifically address.

\section{Conclusion}

The general framework of HAFM with its modules presented in this study includes common practices for practitioners recommended by various pre-existing guidelines. The main purpose of proposing this framework is to make it more 
explicit and transparent so that its related tasks, including professional services, administration, and financial aspects can be systematized without creating any misunderstandings among stakeholders.

Explicitly systemizing the framework of HAFM provides substantial benefits of engaging with various stakeholders: hearing aid users, service providers, manufacturers, and public health administrators. For instance, by modularizing the whole procedure of HAFM, service providers systematically identify and administer comprehensive components and outlines of HAFM leading to the improved service quality and user satisfaction. This contributes to enhance patient-centered services, patient' self-efficacy, and adherence rates with hearing aids (e.g., consistently using hearing aids and attending follow-up appointments). Additionally, systematized services with this framework improve sound quality and comfort of hearing aid users. As a result, it contributes to increase hearing aid adoption rates or decrease hearing aid return rates. Above this, the cost calculation based on each module or each component is applicable to improve public health funding system for the people with hearing impairments as well as clarify payment claim. Another possible application is to develop professional training programs of audiology students and hearing health providers as well as experts in HAFM. This framework based on existing guidelines for hearing aid fitting provides the first insight toward the international standardization of HAFM.

\section{Acknowledgments}

This work was supported by a grant from the Korean Ministry of Trade, Industry \& Energy, Project No. 10049456.

\section{Conflicts of interest}

The authors have no financial conflicts of interest.

\section{REFERENCES}

1) WHO global estimates on prevalence of hearing loss. Geneva: World Health Organization;2012. Available from: http://www.who.int/pbd/ deafness/estimates.

2) WHO. Millions of people in the world have hearing loss that can be treated or prevented. Geneva: WHO;2013. p.1-17.

3) Hougaard S, Ruf S. EuroTrak I. A consumer survey about hearing aids in Germany, France and the UK. Hear Rev 2011;18:12-28.

4) Strom KE. Hearing aid sales increase by $4.8 \%$ in 2014 ; RICs continue market domination. Hear Rev 2015;22:6.

5) Consumer Reports. Hear well in noisy world. Available from: http:// www.consumerreports.org/cro/magazine-archive/july-2009/health/ hearing-aids/overview/hearing-aids-ov.htm.

6) Kochkin S, Beck DL, Christensen LA, Compton-Conley C, Kricos PB, Fligor BJ, et al. MarkeTrak VIII: The impact of the hearing healthcare professional on hearing aid user success. Hear Rev 2010; 17:12-34.

7) Kochkin S. MarkeTrak VII: Obstacles to adult non-user adoption of hearing aids. Hear J 2007;60:24-50.

8) Moodie S, Scollie S, Seewald R, Bagatto M, Beaulac S. The DSL method for pediatric and adult hearing instrument fitting; version 5 . Phonak Focus 2007;1-22.

9) Schum DJ, Weile JN. Creating a more personalized hearing aid fitting. Oticon White Paper 2003;1-16. Available from: http://www.oticonusa.com/ asset/cache.ashx?id=20728\&type=14\&format=web.

10) Stevens K. A hearing aid fitting protocol. Take the time, Do it right. Innovations 2014;4:18-27.

11) Audiology Australia. Audiology Australia professional practice standards-part B clinical standards. Audiological Society of Australia. Audiology Australia professional practice standards-part B clinical standards. Australia: Audiological Society of Australia;2013. Available from: http://www.audiology.asn.au/standards-downloads/Clinical \%20Standards \%20-\%20whole\%20document \%20July13\%201.pdf.

12) Dillon H. Hearing Aids. 2nd ed. Turramurra: Boomerang Press/ Thieme;2012. p.1-631.

13) British Academy of Audiology. Scope of Practice. Available from: http://www.baaudiology.org/careers/scope-practice/\#.VhNkKjuhdjq.

14) Feirn R, Wood S, Sutton G, Booth R, Meredith R, Brennan S, et al. Guidelines for Fitting Hearing Aids to Young Infants. February 2014. Available from: http://www.psych-sci.manchester.ac.uk/mchas/innfantHAfittingguidelines/infantHAfittingguidelines.pdf.

15) College of Audiologists and Speech-Language Pathologists of Ontario. Preferred practice guideline for the prescription of hearing aids to adults. Available from: http://www.caslpo.com/sites/default/uploads/files/PPG_EN_Prescriptions_Hearing_Aids_Adults.pdf.

16) EN 15927 Services offered by hearing aid professionals. Belgium: European Standard;2010.

17) AEA. Resolutions and recommendations adopted in the General Assembly. Available from: http://www.aea-audio.org/portal/index. $\mathrm{php} /$ resolutions/item/158-resolutions-and-recommendations-adopted-in-the-general-assembly/158-resolutions-and-recommendationsadopted-in-the-general-assembly?start $=5$.

18) EUHA. 10 step catalogue on the quality of hearing aid fitting. European Union of Hearing Aid Acousticians. Available from: http:// www.euha.org/information/.

19) ISA. Good Practice Guidance for Adult Hearing Aid Fittings and Services - Background to the Document and Consultation. Available from: http://www.isa-audiology.org/members/pdf/gpg-adaf.pdf.

20) New Zealand Audiological Society. Hearing aid selection, fitting and follow-up. Available from: http://www.audiology.org.nz/userfiles/file/pdf/Hr\%20Aid\%20Slction.pdf.

21) Lee JH, Lee KW. Hearing aid evaluation. Seoul: Hakjisa;2005. p.1-397.

22) ASHA Ad Hoc Committee on Hearing Aid Selection and Fitting. Guidelines for Hearing Aid Fitting for Adults. Am J Audiol 1998;7: $5-13$.

23) Valente M, Abrams H, Benson D, Chisolm T, Citron D, Hampton D, et al. American Academy of Audiology guidelines for the audiologic management of adult hearing loss. Audiol Today 2006;18:32-6.

24) American Academy of Audiology. American Academy of Audiology Clinical Practice Guidelines. Pediatric amplification. Available from: http://galster.net/wp-content/uploads/2013/07/AAA-2013-Pediatric-Amp-Guidelines.pdf.

25) American Academy of Audiology. Adult patients with servere to profound unilateral sensorineural hearing loss. Available from: http:/ www.audiology.org/sites/default/files/PractGuidelineAdultsPatients WithSNHL.pdf.

26) World Health Organization. Guidelines for hearing aids and services for developing countries. Geneva: World Health Organization;2004. p.1-38.

27) ISO. ISO/IEC 19501:2005(en). Information technology - Open Distributed Processing - Unified Modeling Language (UML) Version 1.4.2. Geneva: ISO;2005.

28) IEC. IEC 60118-7:2005. Electroacoustics - Hearing aids - Part 7: Measurement of the performance characteristics of hearing aids for production, supply and delivery quality assurance purposes. Geneva: 
Oh SH, et al.

IEC;2005.

29) ISO. ISO 12124:2001. Acoustics -- Procedures for the measurement of real-ear acoustical characteristics of hearing aids. Geneva: ISO; 2001.

30) ISO. ISO 8253-2:2009. Acoustics -- Audiometric test methods --
Part 2: Sound field audiometry with pure-tone and narrow-band test signals. Geneva: ISO;2009.

31) Humes LE. Dimensions of hearing aid outcome. J Am Acad Audiol 1999;10:26-39. 